\title{
Circular Truncated Conical Mirror with Cavity Shape and Inner Reflection for Concentrating Diffused Light to Collector Mouth from a Half Celestial Sphere
}

\author{
Mitsuhiro Matsumoto* ${ }^{* \dagger}$ Member
}

(Received November 27, 2020, revised January 16, 2021)

\begin{abstract}
In this study, we designed a circular truncated conical mirror to concentrate diffused light to the collector mouth from a half celestial sphere and evaluated its performance. The conical mirror has a circular truncated cone-shaped cavity, which reflects light on the cavity's inner surface. The top of the truncated cone shape is the incident mouth, the bottom is the collector mouth, and the sides are the mirror surfaces. The incident mouth size is smaller than the collector mouth size. All light incident on the incident mouth is either reflected or not reflected on the mirror surface to reach the collector mouth. Light incident at a shallow angle to the central axis of the conical mirror is not reflected on the mirror surface, while light incident at a deep angle is reflected on the mirror surface. The conical mirror can concentrate all diffused light incident at the incident mouth from a half celestial sphere of $360^{\circ}$ in the horizontal direction and $180^{\circ}$ in the vertical direction to the collector mouth. A coordinate system is established for the conical mirror, and a design method is proposed. Using our design method, the conical mirror is manufactured from the determined design values. A plane mirror, concave mirror, compound parabolic mirror, and Fresnel lens, comprising the conical mirror, are used as the measured objects to compare the manufactured conical mirror performance. An experimental setup is constructed to evaluate the performance of the measured objects. Using the constructed experimental setup, diffused light is irradiated to the measured objects as scattered light. The illuminance of the light concentrated in the collector mouth of the measured objects is measured for the irradiated diffused light. The measured illuminance is then used to compare and evaluate the light-concentrating efficiency of the measured objects.
\end{abstract}

Keywords: Circular Truncated Conical Mirror, Cavity Shape, Inner Reflection, Light Concentration, Diffused Light, Collector Mouth, Half Celestial Sphere, Illuminance

\section{Introduction}

A concentrating-type thermal collector that converts light energy into heat energy by concentrating direct light from the sun using a light concentrator, which directs the light onto the thermal collector body at the collector mouth is widely used [1]. Plane, concave, and compound parabolic mirrors, as well as Fresnel lenses are used as light concentrators [1]. However, such concentrating-type thermal collectors can only concentrate direct sunlight and are unable to concentrate scattered diffuse light from the entire sky, which is a half celestial sphere [2]. The proportion of the scattered light is high in most regions of the world, except in regions with year-round fine weather, such as desert areas [2]. In Japan, approximately half of the annual total solar radiation is the scattered light [2]. Especially in the Sea of Japan side from San'in to Hokkaido, there are more than 180 days with an average cloud cover of 8.5 or more in which only scattered light illuminates [3]. Therefore, a

\footnotetext{
* Corresponding: m-matsumoto@kanagawa-u.ac.jp

$\dagger$ Faculty of Engineering, Kanagawa University, 3-27-1, Rokkakubashi, Kanagawa-ku, Yokohama-shi, Kanagawa, 221-8686, Japan
}

light concentrator that can concentrate diffused light such as scattered light from a half celestial sphere to the collector mouth is required in places such as the Sea of Japan.

Studies have been conducted on concentrators to concentrate scattered light. Winston, Derrick et al. and Rao et al. used a parallel arrangement of compound parabolic mirrors [4] [6]. Mather et al. and Mills et al. used symmetric and asymmetric cylindrical-shaped mirrors [7] [8]. Mather et al. and Derrick et al. used involute-shaped mirrors [5] [7]. Bassett et al. used symmetrical-shaped and asymmetrical-shaped mirrors [9]. Grimmer, Frissora et al., Norton et al., Suzuki, Eames et al. and Wang et al. used both symmetrical- and asymmetrical-shaped compound parabolic mirrors [10] [16]. Grimmer and Derrick et al. used parabolic mirrors [5] [10]. Derrick et al. used plane mirrors [5]. Yamada et al. used Fresnel lenses, cylindrical lenses, water cylindrical lenses, and parabolic mirrors [17]. However, all studies have only demonstrated two-dimensional light concentrators, which is insufficient to concentrate diffused light from the three-dimensional half celestial sphere. Research is being conducted on threedimensional concentrators. Sellami et al. demonstrated 
square elliptic hyperbolic mirrors [18]. However, the square elliptical hyperbolic mirrors only concentrate direct light onto solar cells. Matsumoto showed polygonal mirrors wherein elliptical mirrors are arranged [19]. However, these polygonal mirrors have a low light-concentrating efficiency.

In this study, a truncated conical mirror having a truncated cone-shaped cavity that reflects light on the inner surface is designed to concentrate the diffused light to the collector mouth from the half celestial sphere, and its performance is evaluated. A coordinate system is established for the conical mirror, and a design method is shown. Using the design method, a conical mirror was manufactured from the determined design values. Plane, concave, and compound parabolic mirrors, and a Fresnel lens, were used as measured objects to compare with the performance of the conical mirror. An experimental setup was constructed to evaluate the performance of the measured objects. Using the constructed experimental setup, diffused light irradiated the measured objects as scattering light. The illuminance of the light concentrated in the collector mouth of each of the measured objects was measured relative to the irradiated diffused light. From the measured illuminance, the light-concentrating efficiency of the measured objects was compared and evaluated.

\section{Mechanism}

Figure 1 shows the mechanism of a conical mirror. Fig. 1(a) shows the plan view, 1(b) shows the front view, and 1(c) shows the bottom view. The conical mirror has a truncated cone-shaped cavity inside a cylindrical body. The cylindrical shape and the truncated cone shape are aligned by their respective central axis, top surface, and bottom surface. The conical mirror then becomes axially symmetric to the central axis. The top of the truncated cone shape is the incident mouth, the bottom is the collector mouth, and the sides are the mirror surfaces. The shapes of the incident mouth and the collector mouth are circular. The size of the incident mouth is smaller than that of the collector mouth. All light incident on the incident mouth is not reflected on the mirror surface or reflected on the mirror surface to reach the collector mouth. The light incident at a shallow angle to the central axis is not reflected on the mirror surface, and the light incident at a deep angle is reflected at the reflection point on the mirror surface. The conical mirror can concentrate all diffused light incident in the incident mouth from a half celestial sphere of $360^{\circ}$ in the horizontal direction and $180^{\circ}$ in the vertical direction, to the collector mouth. For example, the vertical direction of the compound parabolic mirror is $90^{\circ}$.

\section{Design}

Table 1 shows a list of variables used herein. A coordinate system was established to design a conical mirror. Figure 2 shows the coordinate system and the design method of the conical mirror. Fig. 2(a) shows the plan view, 2(b) shows the front view, and 2(c) shows the bottom view. The coordinate system of the conical mirror is an $\mathrm{R}-\Theta-\mathrm{Z}$ coordinate system. In the R- $\Theta-Z$ coordinate system, the center of the

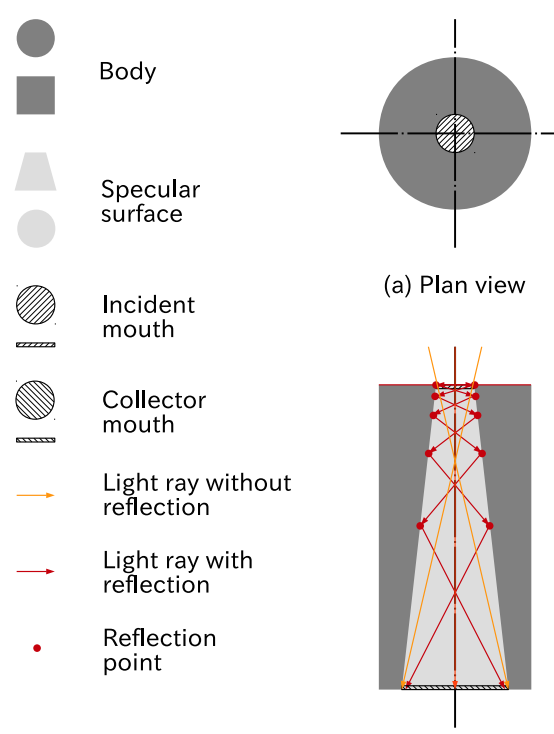

(b) Front view

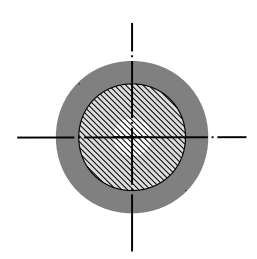

(c) Bottom view

Figure 1: Mechanism of conical mirror at plan view, front view and bottom view.

incident mouth is set as origin $\mathrm{O}$, the radial direction of the incident mouth as R-axis, the direction of rotation with the center axis as axis as $\Theta$-axis, and the direction of the collector mouth from the incident mouth on the center axis as $\mathrm{Z}$-axis. The incident mouth and the collector mouth are on a plane perpendicular to the $\mathrm{Z}$-axis and parallel to the $\mathrm{R}-\Theta$ plane of the $\mathrm{R}-\Theta-\mathrm{Z}$ coordinate system. Let I be the endpoint of the incident mouth and $\mathrm{C}$ be the endpoint of the collector mouth. Let $r_{\mathrm{I}}$ be the radius in the R-axis direction at the incident mouth, $r_{\mathrm{C}}$ be the radius in the R-axis direction at the collector mouth, and $h$ be the height in the $\mathrm{Z}$-axis direction in the conical mirror. Using radii $r_{\mathrm{I}}$ and $r_{\mathrm{C}}$, the diameter of the incident mouth becomes $2 r_{\mathrm{I}}$ and the diameter of the collector mouth becomes $2 r_{\mathrm{C}}$. The area of the incident mouth is $a_{\mathrm{I}}$, and the area of the collector mouth is $a_{\mathrm{C}}$. The incident mouth is a circle with radius $r_{\mathrm{I}}$ centered at origin $\mathrm{O}$ and has an area $a_{\mathrm{I}}$. The collector mouth is a circle with radius $r_{\mathrm{C}}$ centered on the Z-axis and has an area $a_{\mathrm{C}}$. The area $a_{\mathrm{I}}$ is smaller than the area $a_{\mathrm{C}}$. Therefore, the radius $r_{\mathrm{I}}$ is smaller than the radius $r_{\mathrm{C}}$. The area $a_{\mathrm{I}}$ can be expressed as

$$
a_{\mathrm{I}}=\pi r_{\mathrm{I}}^{2}
$$

The area $a_{\mathrm{C}}$ can be expressed as

$$
a_{\mathrm{C}}=\pi r_{\mathrm{C}}^{2}
$$

The shape of the conical mirror is axially symmetric to the $\mathrm{Z}$-axis, which is the central axis. Therefore, subsequently, 
the conical mirror is designed on the R-Z plane.

In the conical mirror, light is incident on the incident mouth from the half celestial sphere centered about the origin $\mathrm{O}$ to the negative direction of the $\mathrm{Z}$-axis from the origin O. All light incident on the incident mouth is not reflected on the mirror surface or reflected on the mirror surface to reach the collector mouth. In the conical mirror, the range in which the light is not reflected on the mirror surface or reflected on the mirror surface is determined. The specular surface where a light ray is reflected in the conical mirror becomes the specular line on the R-Z plane. The specular line is placed symmetrically to the $\mathrm{Z}$-axis in the first and fourth quadrants. Let $\phi$ be the angle between the Z-axis and the light ray. Let $\phi_{\mathrm{L}}$ be the angle between the Z-axis and the light ray passing through the endpoints I and $\mathrm{C}$. The coordinates of the endpoint I on the R-Z plane are $\left(r_{\mathrm{I}}, 0\right)$ and the coordinates of the endpoint $\mathrm{C}$ are $\left(-r_{\mathrm{C}}, h\right)$. Let $\phi_{\mathrm{R}}$ be the angle between the $\mathrm{Z}$-axis and the specular line of the first quadrant, or between the $\mathrm{Z}$-axis and the specular line of the fourth quadrant. The angles $\phi_{\mathrm{L}}$ and $\phi_{\mathrm{R}}$ are obtained from the relation of trigonometric function using radius $r_{\mathrm{I}}$, radius $r_{\mathrm{C}}$ and height $h$. From height $h$, and sum of the radii $r_{\mathrm{C}}$ and $r_{\mathrm{I}}$, the angle $\phi_{\mathrm{L}}$ can be expressed as

$$
\tan \phi_{\mathrm{L}}=\frac{r_{\mathrm{C}}+r_{\mathrm{I}}}{h} \quad\left(r_{\mathrm{C}}>r_{\mathrm{I}}\right)
$$

Using height $h$ and the value obtained by subtracting the radius $r_{\mathrm{I}}$ from the radius $r_{\mathrm{C}}$, the angle $\phi_{\mathrm{R}}$, can be expressed as

$$
\tan \phi_{\mathrm{R}}=\frac{r_{\mathrm{C}}-r_{\mathrm{I}}}{h} \quad\left(r_{\mathrm{C}}>r_{\mathrm{I}}\right)
$$

Using Equations (3) and (4), the range of angle $\phi$ not reflected on the specular line can be expressed as

$$
-\phi_{\mathrm{L}} \leqq \phi \leqq \phi_{\mathrm{R}}
$$

Using Equations (3) and (4), the range of angle $\phi$ reflected on the specular line can be expressed as

$$
\begin{aligned}
& -\frac{\pi}{2} \leqq \phi<-\phi_{\mathrm{L}} \\
& \phi_{\mathrm{R}}<\phi \leqq \frac{\pi}{2}
\end{aligned}
$$

In the conical mirror, the light incident at a small and shallow angle $\phi$ is not reflected on the specular surface, but the light incident at a large and deep angle $\phi$ is reflected on the specular surface with respect to the central axis, which is the $\mathrm{Z}$-axis. In the design, we consider how many times the light with a large angle $\phi$ is reflected on the specular line to reach the collector mouth. Onizuka demonstrated that the light incident on the edge of the incident mouth is most often reflected on the specular surface before reaching the collector mouth [20]. He also demonstrated that when the angle of incidence of the light on the specular surface is large, the light is most reflective by the time it reaches the collector mouth [20]. Therefore, at endpoint I, the light incident at the largest angle $\phi$ of $90^{\circ}$, from the R-axis direction, is most reflected on the specular line and reaches the collector mouth. Under these conditions, the radius $r_{\mathrm{I}}$, radius
$r_{\mathrm{C}}$, and height $h$ are determined for the number of times the light is reflected on the specular line. Let the coordinates on the $\mathrm{R}-\mathrm{Z}$ plane be $(r, z)$. The equation of the specular line can be expressed using the radius $r_{\mathrm{I}}$ and the obtained angle $\phi_{R}$. The equation of the specular line in the first quadrant has a slope of $\tan \phi_{\mathrm{R}}$ and an intercept of radius $r_{\mathrm{I}}$. Using Equation (4), $z$ can be expressed as

$$
z=\tan \phi_{\mathrm{R}} r+r_{\mathrm{I}}
$$

The equation of the specular line in the fourth quadrant has a slope of $\tan \left(-\phi_{\mathrm{R}}\right)$ and an intercept of radius $-r_{\mathrm{I}}$. Using Equation (4), $z$ can be expressed as

$$
z=\tan \left(-\phi_{\mathrm{R}}\right) r-r_{\mathrm{I}}
$$

Let $t$ be the turn in which light reflects off the specular line. The turn $t$ is a natural number, including 0 . Moreover, let the natural number, including 0 , be $n$. Let the reflection point at turn $t$ be $\mathrm{R}_{t}$. Let the coordinates of the reflection point $\mathrm{R}_{t}$ be $\left(r_{\mathrm{R} t}, z_{\mathrm{R} t}\right)$. Let the angle between the $\mathrm{Z}$-axis and the reflected ray is $\phi_{\mathrm{R} t}$ at the reflection point $\mathrm{R}_{t}$. The reflection point $\mathrm{R}_{1}$, whose turn $t$ is 1 , coincides with the endpoint I. In the design, the reflection point $\mathrm{R}_{1}$, whose turn $t$ is 1 , is set on the specular line in the first quadrant. The turn $t$ in which light is reflected by the specular line in the first quadrant can be expressed, using the natural number $n$, as

$$
t=2 n+1 \quad(n=0,1,2, \cdots)
$$

The turn $t$ in which light is reflected by the specular line in the fourth quadrant can be expressed, using the natural number $n$, as

$$
t=2 n \quad(n=0,1,2, \cdots)
$$

The angle $\phi_{\mathrm{R} t}$ can be expressed using the angle $\phi_{\mathrm{R} t-1}$ and the obtained angle $\phi_{\mathrm{R}}$. Let the angle $\phi_{\mathrm{R} 0}$ be $90^{\circ}$, which is the largest in the half celestial sphere. As the light ray is specularly reflected at the reflection point $\mathrm{R}_{t}$, the angle of incidence and the angle of reflection of the light ray at the reflection point $\mathrm{R}_{t}$ are equal. Therefore, the angle $\phi_{\mathrm{R} t}$ in the first quadrant can be expressed using Equations (4) and (9) as

$$
\phi_{\mathrm{R} t}=2 \phi_{\mathrm{R}}-\phi_{\mathrm{R} t-1} \quad\left(\phi_{\mathrm{R} 0}=\frac{\pi}{2}\right)
$$

The angle $\phi_{\mathrm{R} t}$ in the fourth quadrant can be expressed using Equations (4) and (10) as

$$
\phi_{\mathrm{R} t}=-2 \phi_{\mathrm{R}}-\phi_{\mathrm{R} t-1}
$$

The equation of the light ray can be expressed using the calculated angle $\phi_{\mathrm{R} t}$, the coordinate $r_{\mathrm{R} t}$, and the coordinate $z_{\mathrm{R} t}$. The coordinate $r_{\mathrm{R} 1}$ can be expressed as radius $r_{\mathrm{I}}$ and the coordinate $z_{\mathrm{R} 1}$ can be expressed as 0 . The equation of the light ray passes through the coordinates $\left(r_{\mathrm{R} t}, z_{\mathrm{R} t}\right)$ of the reflection point $\mathrm{R}_{t}$ with a slope of $\tan \phi_{\mathrm{R} t}$. The reflection point $\mathrm{R}_{t}$ in the first quadrant can be expressed using the Equation 
Table 1: List of variables used in equations.

\begin{tabular}{cc|cc}
\hline Variable & Meaning & Variable & Meaning \\
\hline$a_{\mathrm{C}}$ & Area of collector mouth & $i_{\mathrm{O}}$ & Illuminance of only lighted object \\
$a_{\mathrm{I}}$ & Area of incident mouth & $i_{\mathrm{S}}$ & Illuminance of light-shielded object \\
$e_{\mathrm{C}}$ & Concentration ratio & $m$ & Maximum number of reflections \\
$e_{\mathrm{I}}$ & Illuminance ratio & $r$ & Natural number \\
$\phi$ & Angle from Z-axis & $r_{\mathrm{C}}$ & Coordinate in R- $\Theta-\mathrm{Z}$ \\
$\phi_{\mathrm{L}}$ & Angle between Z-axis and light ray on I and C & $r_{\mathrm{I}}$ & Radius of collector mouth \\
$\phi_{\mathrm{R}}$ & Angle between Z-axis and specular line & $r_{\mathrm{R} t}$ & Coordinate of $\mathrm{R}_{t}$ \\
$\phi_{\mathrm{R} t}$ & Angle between Z-axis and light ray reflected at $\mathrm{R}_{t}$ & $t$ & Turn of reflection \\
$h$ & Height & $z^{2}$ & Coordinate in R- $\Theta-\mathrm{Z}$ \\
$i_{\mathrm{C}}$ & Illuminance of dome light in collector mouth area & $z$ & Coordinate of $\mathrm{R}_{t}$ \\
$i_{\mathrm{I}}$ & Illuminance of dome light in incident mouth area & $z_{\mathrm{R} t}$ & - \\
$i_{\mathrm{L}}$ & Illuminance of lighted object & - & \\
\hline
\end{tabular}

(11), and in the fourth quadrant can be expressed using the Equation (12) as

$$
z=\tan \phi_{\mathrm{R} t} r+z_{\mathrm{R} t}-\tan \phi_{\mathrm{R} t} r_{\mathrm{R} t} \quad\left(r_{\mathrm{R} 1}=r_{\mathrm{I}}, z_{\mathrm{R} 1}=0\right)
$$

For the light incident at the reflection point $\mathrm{R}_{1}$ which is the endpoint $\mathrm{I}$, the turn $t$ is sequentially increased from 1 , and the angle $\phi_{\mathrm{R} t}$ indicating the direction of the light reflected at the reflection point $\mathrm{R}_{t}$ is calculated. The coordinates $\left(r_{\mathrm{R} t}, z_{\mathrm{R} t}\right)$ of the reflection point $\mathrm{R}_{t}$ are obtained using the equation of the specular line shown in Equations (7) and (8), and the equation of the light ray shown in Equation (13). The values of radii $r_{\mathrm{I}}$ and $r_{\mathrm{C}}$ are fixed, the value of height $h$ is changed, and the coordinates $\left(r_{\mathrm{R} t}, z_{\mathrm{R} t}\right)$ are calculated. Let $m$ be the maximum number of times the light traveling in the direction of the collector mouth is reflected by the specular line before reaching the collector mouth. Consider the reflection points $\mathbf{R}_{m}$ and $\mathbf{R}_{m+1}$ across the height $h$ for the number $m$. The conditions for completing the calculation are that the coordinate $z_{\mathrm{R} m}$ is less than the height $h$ when absolute value of the coordinate $r_{\mathrm{R} m}$ is less than the radius $r_{\mathrm{C}}$, for the light reflected at the reflection points $\mathbf{R}_{m}$ and $\mathbf{R}_{m+1}$ and that the coordinate $z_{\mathrm{R} m}$ is greater than height $h$ when absolute value of the coordinate $r_{\mathrm{R} m+1}$ is greater than the radius $r_{\mathrm{C}}$. The conditions for completing the calculation can be expressed as

$$
\begin{aligned}
& z_{\mathrm{R} m}<h \quad\left(\left|r_{\mathrm{R} m}\right|<r_{\mathrm{C}}\right) \\
& z_{\mathrm{R} m+1} \geqq h \quad\left(\left|r_{\mathrm{R} m+1}\right| \geqq r_{\mathrm{C}}\right)
\end{aligned}
$$

The concentration ratio is obtained to evaluate the concentrating performance of the designed conical mirror. Let the concentration ratio be $e_{\mathrm{C}}$. The concentration ratio $e_{\mathrm{C}}$ is the ratio of the area $a_{\mathrm{I}}$ to the area $a_{\mathrm{C}}$. The concentration ratio $e_{\mathrm{C}}$ can be expressed using Equations (1) and (2) as

$$
e_{\mathrm{C}}=\frac{a_{\mathrm{I}}}{a_{\mathrm{C}}}
$$

\section{Performance evaluation}

4.1 Manufacturing the conical mirror Table 2 shows the design values of the conical mirror. A conical mirror whose number $m$ is from 1 to 5 was designed, and the effect of the number $m$ on the concentrating performance

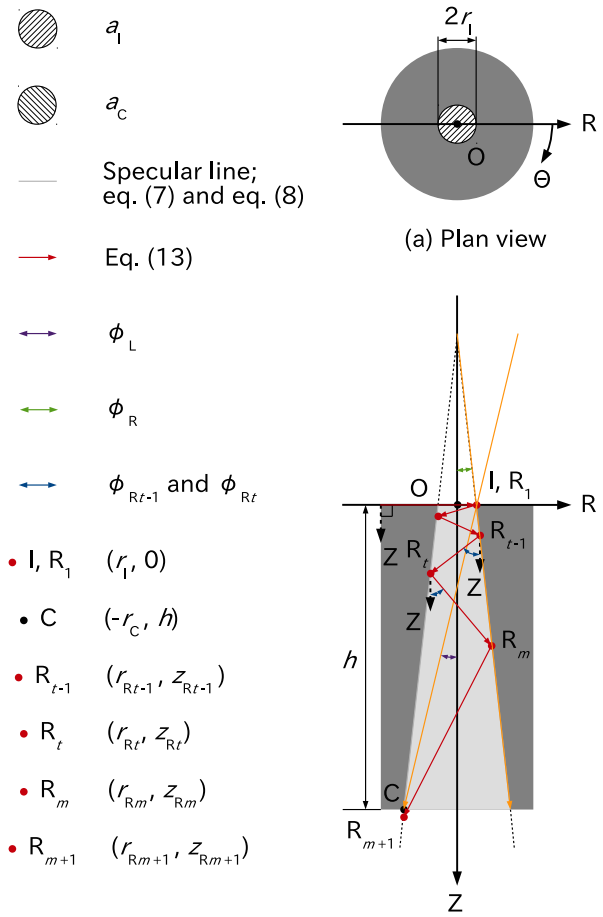

(b) Front view

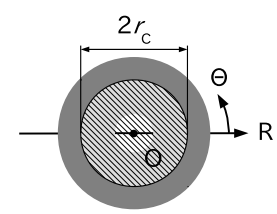

(c) Bottom view

Figure 2: Coordinate system and design method of conical mirror at plan view, front view and bottom view.

was revealed. Conical mirrors with radii $r_{\mathrm{C}}$ of $7 \mathrm{~mm}$ and $4.9 \mathrm{~mm}$ were designed, and the effect of the radius $r_{\mathrm{C}}$ on the concentrating performance was clarified. The radius $r_{\mathrm{I}}$ was based on the size of the compound parabolic mirror to compare it with the compound parabolic mirror shown below. The radius $r_{\mathrm{C}}$ was based on the size of the light receiver shown below and the intermediate size between the light receiver and the compound parabolic mirror. The height $h$ 
Table 2: Design values of the maximum number of reflections, radius of incident mouth, radius of collector mouth, height, angle between Z-axis and light ray on I and C, angle between Z-axis and specular line, area of incident mouth, area of collector mouth and concentration ratio in the conical mirror.

\begin{tabular}{ccccccccc}
\hline$m$ & $r_{\mathrm{I}}[\mathrm{mm}]$ & $r_{\mathrm{C}}[\mathrm{mm}]$ & $h[\mathrm{~mm}]$ & $\phi_{\mathrm{L}}[\mathrm{rad}]$ & $\phi_{\mathrm{R}}[\mathrm{rad}]$ & $a_{\mathrm{I}}\left[\mathrm{mm}^{2}\right]$ & $a_{\mathrm{C}}\left[\mathrm{mm}^{2}\right]$ & $e_{\mathrm{C}}$ \\
\hline 1 & 2.7 & 7 & 10 & 0.77 & 0.406 & 22.9 & 153.9 & 0.148 \\
2 & 2.7 & 7 & 17.7 & 0.501 & 0.238 & 22.9 & 153.9 & 0.148 \\
3 & 2.7 & 7 & 25.2 & 0.367 & 0.169 & 22.9 & 153.9 & 0.148 \\
4 & 2.7 & 7 & 32.6 & 0.289 & 0.131 & 22.9 & 153.9 & 0.148 \\
5 & 2.7 & 7 & 40 & 0.237 & 0.107 & 22.9 & 153.9 & 0.148 \\
1 & 2.7 & 4.9 & 6.1 & 0.894 & 0.346 & 22.9 & 75.4 & 0.303 \\
\hline
\end{tabular}

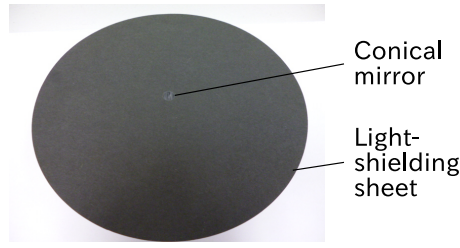

(a) With specular surface

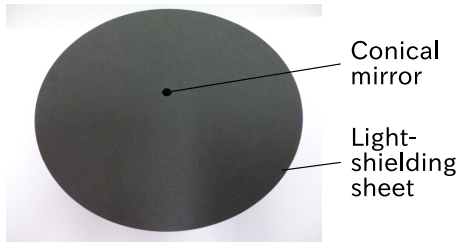

(b) Without specular surface

Figure 3: Images of the conical mirrors with specular surface, and without specular surface, and the light-shielding sheet in the two conical mirrors.

was determined from the design calculation results shown in Tables 3 to 8. The angle $\phi_{\mathrm{L}}$ uses Equation (3), angle $\phi_{\mathrm{R}}$ uses Equation (4), area $a_{\mathrm{I}}$ uses Equation (1), area $a_{\mathrm{C}}$ uses Equation (2), and concentration ratio $e_{\mathrm{C}}$ uses Equation (15) to determine each design value.

Tables 3 to 8 show the design calculation results of the conical mirror. Table 3 shows the case when $m=1$ and radius $r_{\mathrm{C}}=7 \mathrm{~mm}$, Table 4 shows the case when $m=2$ and radius $r_{\mathrm{C}}=7 \mathrm{~mm}$, Table 5 shows the case when $m=3$ and radius $r_{\mathrm{C}}=7 \mathrm{~mm}$, Table 6 shows the case when $m$ is 4 and radius $r_{\mathrm{C}}=7 \mathrm{~mm}$, Table 7 shows the case when $m=5$ and radius $r_{\mathrm{C}}=7 \mathrm{~mm}$, and Table 8 shows the case when $m=1$ and radius $r_{\mathrm{C}}=4.9 \mathrm{~mm}$. The coordinates $r_{\mathrm{R} t}$ and $z_{\mathrm{R} t}$, and the angle $\phi_{\mathrm{R} t}$ are calculated each for turn $t$ of 1-2 in Tables 3 and 8 , for turn $t$ of 1-3 in Table 4, for turn $t$ of 1-4 in Table 5, for turn $t$ of 1-5 in Table 6, and for turn $t$ of 1-6 in Table 7, using Equations (7) to (13). The condition shown in Equation (14) is satisfied for turn $t$ of 1-2 in Tables 3 and 8 , for turn $t$ of 2 and 3 in Table 4 , for turn $t$ of 3 and 4 in Table 5 , for turn $t$ of 4 and 5 in Table 6 , and for turn $t$ of 5 and 6 in Table 7.

Conical mirrors with and without a specular surface were manufactured to evaluate the effect of reflection on the specular surface. Figure 3 shows the images of the conical mirrors. Fig. 3(a) shows the conical mirror with a specular surface, and Fig. 3(b) shows the conical mirror without a specular surface. The conical mirrors were manufactured using the design values shown in Table 2 . The body is a black ABS resin. For the conical mirror with specular surface, Viva mirror film from Takahara Corporation was used for the specular surface. The Viva mirror film is a film formed by applying a $0.06-\mathrm{mm}$-thick protective coat on to a 0.1 mm-thick aluminum-deposited PET sheet. The reflectance of aluminum in visible light is approximately $90 \%$. For the
Table 3: The turn of reflection, coordinates of $\mathrm{R}_{t}$, and angle between $\mathrm{Z}$-axis and light ray reflected at $\mathrm{R}_{t}$ in the designed conical mirror when $m$ is 1 , and $r_{\mathrm{C}}$ is $7 \mathrm{~mm}$.

\begin{tabular}{cccc}
\hline$t$ & $r_{\mathrm{R} t}[\mathrm{~mm}]$ & $z_{\mathrm{R} t}[\mathrm{~mm}]$ & $\phi_{\mathrm{R} t}[\mathrm{rad}]$ \\
\hline 1 & 2.7 & 0 & -0.758 \\
2 & -7.1 & 10.4 & -0.053 \\
\hline
\end{tabular}

Table 4: The turn of reflection, coordinates of $\mathrm{R}_{t}$, and angle between $\mathrm{Z}$-axis and light ray reflected at $\mathrm{R}_{t}$ in the designed conical mirror when $m$ is 2 , and $r_{\mathrm{C}}$ is $7 \mathrm{~mm}$.

\begin{tabular}{cccc}
\hline$t$ & $r_{\mathrm{R} t}[\mathrm{~mm}]$ & $z_{\mathrm{R} t}[\mathrm{~mm}]$ & $\phi_{\mathrm{R} t}[\mathrm{rad}]$ \\
\hline 1 & 2.7 & 0 & -1.094 \\
2 & -3.4 & 3.1 & 0.617 \\
3 & 7 & 18 & -0.14 \\
\hline
\end{tabular}

Table 5: The turn of reflection, coordinates of $\mathrm{R}_{t}$, and angle between $\mathrm{Z}$-axis and light ray reflected at $\mathrm{R}_{t}$ in the designed conical mirror when $m$ is 3 , and $r_{\mathrm{C}}$ is $7 \mathrm{~mm}$.

\begin{tabular}{cccc}
\hline$t$ & $r_{\mathrm{R} t}[\mathrm{~mm}]$ & $z_{\mathrm{R} t}[\mathrm{~mm}]$ & $\phi_{\mathrm{R} t}[\mathrm{rad}]$ \\
\hline 1 & 2.7 & 0 & -1.232 \\
2 & -3 & 2 & 0.894 \\
3 & 4 & 7.6 & -0.556 \\
4 & -7 & 25.4 & 0.218 \\
\hline
\end{tabular}

conical mirror without a specular surface, a light-shielding sheet was used instead of a Viva mirror film for the specular surface part. The parts other than the specular surface were covered with the light-shielding sheet.

4.2 Measured objects Table 9 shows the specifications of the measured objects: The plane mirror, concave mirror, compound parabolic concentrator, and Fresnel lens. 
Table 6: The turn of reflection, coordinates of $\mathrm{R}_{t}$, and angle between $\mathrm{Z}$-axis and light ray reflected at $\mathrm{R}_{t}$ in the designed conical mirror when $m$ is 4 , and $r_{\mathrm{C}}$ is $7 \mathrm{~mm}$.

\begin{tabular}{cccc}
\hline$t$ & $r_{\mathrm{R} t}[\mathrm{~mm}]$ & $z_{\mathrm{R} t}[\mathrm{~mm}]$ & $\phi_{\mathrm{R} t}[\mathrm{rad}]$ \\
\hline 1 & 2.7 & 0 & -1.308 \\
2 & -2.9 & 1.5 & 1.046 \\
3 & 3.3 & 5.1 & -0.783 \\
4 & -4.4 & 12.9 & 0.521 \\
5 & 7 & 32.8 & -0.259 \\
\hline
\end{tabular}

Table 7: The turn of reflection, coordinates of $\mathrm{R}_{t}$, and angle between $\mathrm{Z}$-axis and light ray reflected at $\mathrm{R}_{t}$ in the designed conical mirror when $m$ is 5 , and $r_{\mathrm{C}}$ is $7 \mathrm{~mm}$.

\begin{tabular}{cccc}
\hline$t$ & $r_{\mathrm{R} t}[\mathrm{~mm}]$ & $z_{\mathrm{R} t}[\mathrm{~mm}]$ & $\phi_{\mathrm{R} t}[\mathrm{rad}]$ \\
\hline 1 & 2.7 & 0 & -1.356 \\
2 & -2.8 & 1.2 & 1.142 \\
3 & 3.1 & 3.9 & -0.928 \\
4 & -3.6 & 9 & 0.714 \\
5 & 4.7 & 18.6 & -0.499 \\
6 & -7 & 40.1 & 0.285 \\
\hline
\end{tabular}

Table 8: The turn of reflection, coordinates of $\mathrm{R}_{t}$, and angle between $\mathrm{Z}$-axis and light ray reflected at $\mathrm{R}_{t}$ in the designed conical mirror when $m$ is 1 , and $r_{\mathrm{C}}$ is $4.9 \mathrm{~mm}$.

\begin{tabular}{cccc}
\hline$t$ & $r_{\mathrm{R} t}[\mathrm{~mm}]$ & $z_{\mathrm{R} t}[\mathrm{~mm}]$ & $\phi_{\mathrm{R} t}[\mathrm{rad}]$ \\
\hline 1 & 2.7 & 0 & -0.878 \\
2 & -5 & 6.3 & 0.186 \\
\hline
\end{tabular}

For comparison with the conical mirror, all incident radii were matched to the radius of the conical mirror, $r_{\mathrm{I}}$. The plane mirror was a 4-6 $\lambda$ plane mirror 9MMAL manufactured by Edmund Optics. The substrate is float glass, and the mirror surface is protected aluminum. The reflectance is more than $85 \%$ on average in the wavelength of light from 400 to $700 \mathrm{~nm}$. The concave mirror was a concave mirror 35X35AL manufactured by Edmund Optics. The substrate is white plate glass, and the mirror surface is protected aluminum. The reflectance is more than $85 \%$ on average in the wavelength of light from 400 to $700 \mathrm{~nm}$. The compound parabolic mirror was a compound parabolic concentrator $45^{\circ} 2.50 \mathrm{MM}$ manufactured by Edmund Optics. The substrate is white plate glass. The transmittance is $90 \%$ or more in the wavelength of light from 400 to $700 \mathrm{~nm}$. The concentration ratio $e_{\mathrm{C}}$ was calculated using Equations (1), (2), and (15), with the radius of the incident mouth as radius $r_{\mathrm{I}}$ and the radius of the collector mouth as radius $r_{\mathrm{C}}$. The Fresnel lens was a Fresnel lens 58X58X38 manufactured by Edmund Optics. The substrate is acrylic. The transmittance is $92 \%$ in the wavelength of light from 400 to $1,100 \mathrm{~nm}$. Figure 4 shows the images of the plane mirror, concave mirror, compound parabolic concentrator, and Fresnel lens. Fig. 4(a) shows the plane mirror, 4(b) shows the concave mirror, 4(c) shows the compound parabolic concentrator, and 4(d) shows the Fresnel lens. The parts other than the incident mouth and exit surface of the plane mirror, concave mirror, compound parabolic concentrator, and Fresnel lens were covered with a light-shielding sheet.

4.3 Experimental setup Figure 5 shows the structure of an experimental setup. Fig. 5(a) shows the plan view, 5(b) shows the front view, and 5(c) shows the bottom view. The experimental setup comprises a dome light, a measured object, and a light receiver. The experimental setup is covered with a light-shielding plate to shield stray light that affects the measurement. An LED light source and a diffuser are installed inside the dome light. The diffuser has the shape of a half celestial sphere which can be generated by rotating a semicircle on the $\mathrm{R}-\mathrm{Z}$ plane of the coordinate system $\mathrm{R}-\Theta-\mathrm{Z}$ around the $\mathrm{Z}$-axis. The equation of the semicircle can be expressed as

$$
r^{2}+z^{2}=40000 \quad(z \leqq 0)
$$

The diffuser can generate diffused light inside the dome light. The measured object and the light receiver align the central axis with the Z-axis. Depending on the measured object to be measured, the measured object and the light receiver are fixed at origin $\mathrm{O}$, point $\mathrm{T}$, and point $\mathrm{B}$ positions on the Z-axis.

Table 10 shows the specifications of the measured objects in the experimental setup. It shows the positions of the light receiver, the light-shielding plate, and the measured objects in the measured object to be measured for the experimental setup shown in Figure 5. The light receiver placed at origin $\mathrm{O}$ and point $\mathrm{B}$ is oriented in the negative direction of the $\mathrm{Z}$-axis, and the light receiver placed at point $\mathrm{T}$ is oriented in the positive direction of the Z-axis. The light-shielding plate was installed for the conical mirror, plane mirror, concave mirror, compound parabolic mirror, and Fresnel lens, all of which were light-shielded. The light-shielded conical mirror and the conical mirror show the incident mouth and the collector mouth in the position of the measured objects, the light-shielded plane mirror and the plane mirror show the specular surface, the light-shielded concave mirror and the concave mirror show the specular surface and the focus point, the light-shielded compound parabolic mirror and the compound parabolic mirror show the incident mouth and the collector mouth, and the light-shielded Fresnel lens and Fresnel lens show the lens surface and the focus point. The specular surface was oriented in the negative direction of the Z-axis. In the light-shielded conical mirror, the light receiver was installed at point $\mathrm{B}$, the light-shielding plate at origin $\mathrm{O}$, the incident mouth at origin $\mathrm{O}$, and the collector mouth at point $\mathrm{B}$. In the light-shielded plane mirror, the light receiver was installed at point $\mathrm{T}$, the light-shielding plate at origin $\mathrm{O}$, and the specular surface at origin $\mathrm{O}$. In the light-shielded concave mirror, the light receiver was installed at point $\mathrm{T}$, the light-shielding plate at origin $\mathrm{O}$, the specular surface at origin $\mathrm{O}$, and the focus point at point $\mathrm{T}$. In the light-shielded compound parabolic mirror, the light receiver was installed at point $\mathrm{B}$, the light-shielding plate at origin $\mathrm{O}$, the incident mouth at origin $\mathrm{O}$, and the exit surface at point B. In the light-shielded Fresnel lens, the light receiver was installed at point $\mathrm{B}$, the light-shielding 
Table 9: The radius of the incident surface, focal length, height, radius of the exit surface, acceptance angle, and concentration ratio in the measured objects (plane mirror, concave mirror, compound parabolic mirror, and Fresnel lens).

\begin{tabular}{ccccccc}
\hline Object name & $\begin{array}{c}\text { Radius of } \\
\text { incident surface } \\
{[\mathrm{mm}]}\end{array}$ & $\begin{array}{c}\text { Focal length } \\
{[\mathrm{mm}]}\end{array}$ & $\begin{array}{c}\text { Height } \\
{[\mathrm{mm}]}\end{array}$ & $\begin{array}{c}\text { Radius of } \\
\text { exit surface } \\
{[\mathrm{mm}]}\end{array}$ & $\begin{array}{c}\text { Acceptance angle } \\
{[\mathrm{rad}]}\end{array}$ & $e_{\mathrm{C}}$ \\
\hline Plane mirror & 2.7 & - & - & - & - & - \\
Concave mirror & 2.7 & 35 & - & - & - & - \\
Compound parabolic mirror & 2.695 & - & 7.52 & 1.25 & 0.785 & 4.64 \\
Fresnel lens & 2.7 & 38.1 & - & - & - & - \\
\hline
\end{tabular}

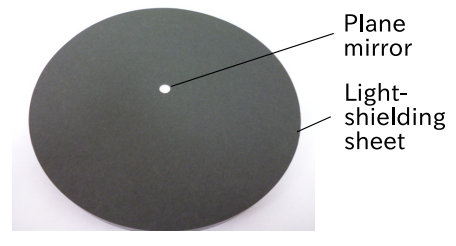

(a) Plane mirror

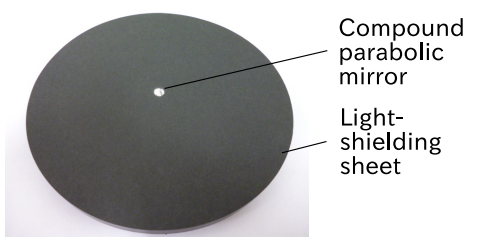

(c) Compound parabolic mirror

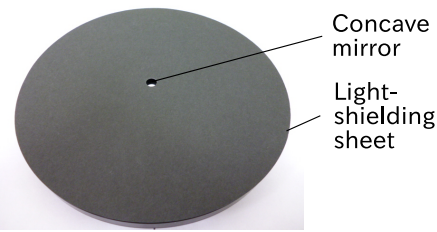

(b) Concave mirror

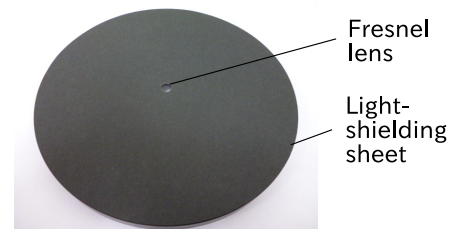

(d) Fresnel lens

Figure 4: Images of the plane, concave, and compound parabolic mirrors, the Fresnel lens, and the light-shielding sheet in the four measured objects.

plate at origin $\mathrm{O}$, the lens surface at origin $\mathrm{O}$, and the focal point at point $\mathrm{B}$. The conical mirror, plane mirror, concave mirror, compound parabolic mirror, and Fresnel lens were the light-shielded conical mirror, light-shielded plane mirror, light-shielded concave mirror, light-shielded compound parabolic mirror, and light-shielded Fresnel lens with the light-shielding plate installed at origin $\mathrm{O}$ removed, respectively.

Figure 6 shows an image of the experimental setup. Fig. 6(a) shows the plan view, 6(b) shows the front view, and 6(c) shows the bottom view. In Fig. 6, the measured object is a conical mirror with a specular surface when $m=5$. The dome light is connected to a power source. The light receiver is connected to an illuminance meter. The HPD2400SW-CR14 light by CCS Inc. was used as the dome light, and PD2-5024 by CCS Inc. was used as the power source. The HPD2-400SW-CR14 generated diffused light by irradiating light on the diffuser plate inside the dome light from the white LED installed inside the dome light. The PD2-5024 could control the dome light in 256 steps by combining 16 steps of coarse dimming and 16 steps of dense dimming. In the experimental setup, coarse dimming was changed as the dimming level, and dense dimming was fixed as one step. T-10MA manufactured by Konica Minolta was used as the light receiver and illuminance meter. The T-10MA could measure the illuminance at the light-receiving surface of the light receiver and display the measured illuminance in the illuminance meter. The lightreceiving surface had a radius of $7 \mathrm{~mm}$ and a light-receiving range in the hemispherical direction. The radius of the lightreceiving surface is matched with the radius $r_{\mathrm{C}}$ of the conical mirror. The range of illuminance that could be measured was 0.01 to $299,9001 x$.

Figure 7 shows an image of diffused light inside the dome light. The dimming level of PD2-5024 was set to 2. The image was taken with a Kodak SP360, which can take pictures horizontally at $360^{\circ}$ and vertically at $214^{\circ}$. The lens of the SP360 was installed at the focal point $\mathrm{O}$ and the lens was oriented in the negative direction of the Z-axis. The diffused light was generated from the diffuser plate at $360^{\circ}$ in the horizontal direction and $180^{\circ}$ in the vertical direction.

4.4 Experimental method Let the illuminance of the dome light in the collector mouth area $a_{\mathrm{C}}$ be $i_{\mathrm{C}}$, the illuminance of the dome light in the incident mouth area $a_{\mathrm{I}}$ be $i_{\mathrm{I}}$, the illuminance of the light-shielded measured object be $i_{\mathrm{S}}$, the illuminance of the measured object to be measured be $i_{\mathrm{L}}$, the illuminance of only measured object be $i_{\mathrm{O}}$, the illuminance ratio indicating the light-concentrating efficiency be $e_{\mathrm{I}}$, and the concentration ratio indicating the light-concentrating efficiency be $e_{\mathrm{C}}$. The illuminance $i_{\mathrm{O}}$ is the value obtained by subtracting the illuminance $i_{\mathrm{S}}$ from the illuminance $i_{\mathrm{L}}$. The illuminance ratio $e_{\mathrm{I}}$ is the ratio of 
Table 10: Specifications of light receiver, light-shielding sheet, incident mouth, mirror surface, incident surface, lens surface, collector mouth, exit surface, and focal point in measured objects of dome light in collector mouth area, dome light in incident mouth area, light-shielded conical mirror, light-shielded plane mirror, light-shielded concave mirror, light-shielded compound parabolic mirror, light-shielded Fresnel lens, conical mirror, plane mirror, concave mirror, compound parabolic mirror, and Fresnel lens in an experimental setup.

\begin{tabular}{cccccc}
\hline $\begin{array}{c}\text { Object } \\
\text { name }\end{array}$ & $\begin{array}{c}\text { Light } \\
\text { receiver }\end{array}$ & $\begin{array}{c}\text { Light-shielding } \\
\text { sheet }\end{array}$ & $\begin{array}{c}\text { Incident mouth, } \\
\text { mirror surface, } \\
\text { incident surface } \\
\text { and lens surface }\end{array}$ & $\begin{array}{c}\text { Collector mouth } \\
\text { and exit surface }\end{array}$ & $\begin{array}{c}\text { Focal } \\
\text { point }\end{array}$ \\
\hline Dome light in collector mouth area & $\mathrm{O}$ & - & - & - & - \\
Dome light in incident mouth area & $\mathrm{O}$ & - & - & - & - \\
Light-shielded conical mirror & $\mathrm{B}$ & $\mathrm{O}$ & $\mathrm{O}$ & $\mathrm{B}$ & - \\
Light-shielded plane mirror & $\mathrm{T}$ & $\mathrm{O}$ & $\mathrm{O}$ & - & - \\
Light-shielded concave mirror & $\mathrm{T}$ & $\mathrm{O}$ & $\mathrm{O}$ & - & $\mathrm{T}$ \\
Light-shielded compound parabolic mirror & $\mathrm{B}$ & $\mathrm{O}$ & $\mathrm{O}$ & $\mathrm{B}$ & - \\
Light-shielded Fresnel lens & $\mathrm{B}$ & $\mathrm{O}$ & $\mathrm{O}$ & - & $\mathrm{B}$ \\
Conical mirror & $\mathrm{B}$ & - & $\mathrm{O}$ & $\mathrm{O}$ & - \\
Plane mirror & $\mathrm{T}$ & - & $\mathrm{O}$ & - & - \\
Concave mirror & $\mathrm{T}$ & - & $\mathrm{O}$ & $\mathrm{T}$ & $\mathrm{T}$ \\
Compound parabolic mirror & $\mathrm{B}$ & - & $\mathrm{O}$ & - & $\mathrm{B}$ \\
Fresnel lens & $\mathrm{B}$ & - & & - \\
\hline
\end{tabular}
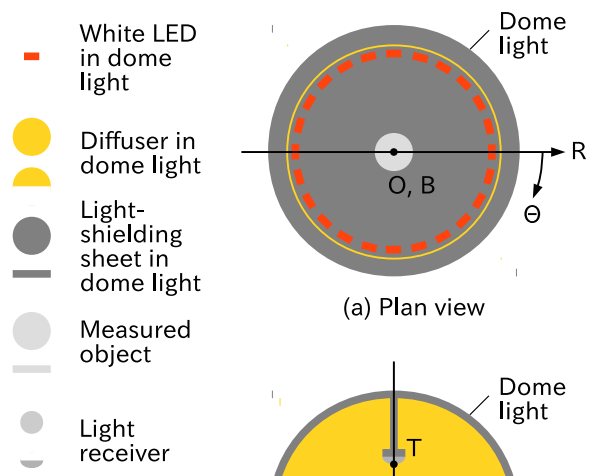

(a) Plan view

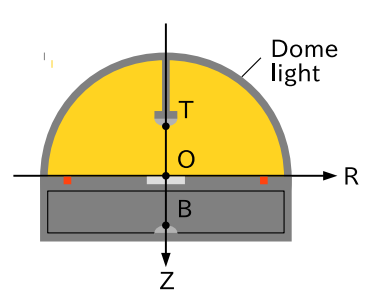

(b) Front view

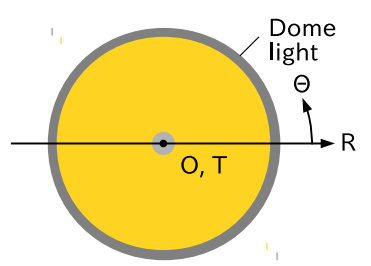

(c) Bottom view

Figure 5: Mechanism of white LED, diffuser, and light-shielding sheet in dome light; measured object and light receiver in experimental setup at plan view, front view and bottom view.

the illuminance $i_{\mathrm{O}}$ to the illuminance $i_{\mathrm{C}}$. The concentration ratio $e_{\mathrm{C}}$ is the ratio of the illuminance $i_{\mathrm{I}}$ to the illuminance $i_{\mathrm{O}}$. The illuminance $i_{\mathrm{O}}$ can be expressed as

$$
i_{\mathrm{O}}=i_{\mathrm{L}}-i_{\mathrm{S}}
$$

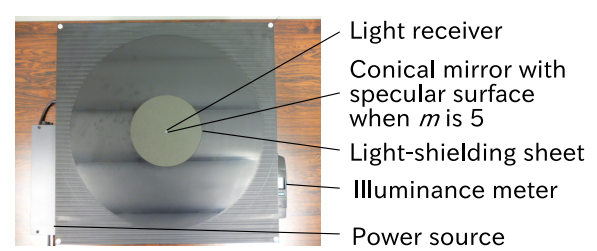

(a) Plan view

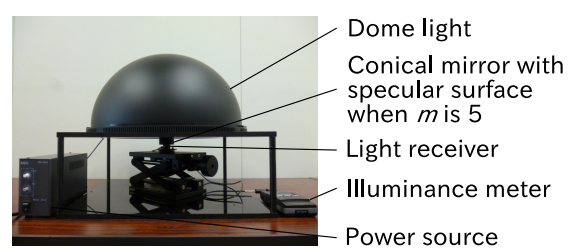

(b) Front view

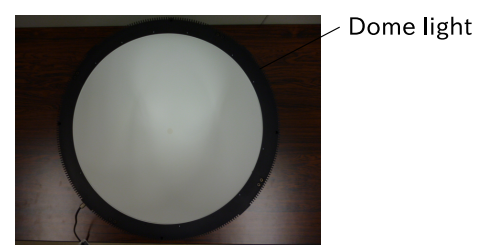

(c) Bottom view

Figure 6: Images of conical mirror with specular surface when $m$ is 5 , light-shielding sheet, illuminance meter, power source, dome light, and light receiver in the experimental setup at plan view, front view, and bottom view.

The illuminance ratio $e_{\mathrm{I}}$ can be expressed using Equation (17) as

$$
e_{\mathrm{I}}=\frac{i_{\mathrm{O}}}{i_{\mathrm{C}}}
$$




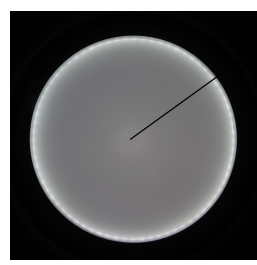

\section{Diffuser}

\section{Light-}

shielding

sheet

Figure 7: Image of light diffused by diffuser and light-shielding sheet in dome light.

The concentration ratio $e_{\mathrm{C}}$ can be expressed using Equation (17) as

$$
e_{\mathrm{C}}=\frac{i_{\mathrm{I}}}{i_{\mathrm{O}}}
$$

The experiment reveals the relation between the dimming level of the dome light and the illuminances $i_{\mathrm{C}}$ and $i_{\mathrm{I}}$. With the dimming level set to 2 , the illuminances $i_{\mathrm{C}}$ and $i_{\mathrm{I}}$ are measured. The illuminances $i_{\mathrm{S}}$ and $i_{\mathrm{L}}$ measured in the measured object are compared. With the dimming level set to 2 , the illuminances $i_{\mathrm{S}}$ and $i_{\mathrm{L}}$ are measured. The illuminance $i_{\mathrm{O}}$ in the measured objects is compared. The illuminance $i_{\mathrm{O}}$ is obtained from the measured illuminances $i_{\mathrm{S}}$ and $i_{\mathrm{L}}$. The illuminance ratio $e_{\mathrm{I}}$ and the concentration ratio $e_{\mathrm{C}}$ in the measured objects are compared. From the obtained illuminances $i_{\mathrm{C}}, i_{\mathrm{I}}$, and $i_{\mathrm{O}}$, the illuminance ratio $e_{\mathrm{I}}$ and the concentration ratio $e_{\mathrm{C}}$ are obtained.

\subsection{Experiment results Figure 8 shows the appear-} ance of the measured objects inside the experimental setup in the experiment. Fig. 8(a) shows the dome light in the collector mouth area, 8 (b) shows the dome light in the incident mouth area, 8(c) shows the light-shielded conical mirror with specular surface when $m$ is $5,8(\mathrm{~d})$ shows the lightshielded conical mirror without specular surface when $m$ is 5, 8(e) shows the light-shielded plane mirror and the lightshielded concave mirror, 8 (f) shows the light-shielded compound parabolic mirror, $8(\mathrm{~g})$ shows the light-shielded Fresnel lens, 8 (h) shows the conical mirror with specular surface when $m$ is 5, 8(i) shows the conical mirror without specular surface when $m$ is $5,8(\mathrm{j})$ shows the plane mirror, $8(\mathrm{k})$ shows the concave mirror, $8(1)$ shows the compound parabolic mirror, and 8(m) shows the Fresnel lens. Fig. 8(a), 8(b), 8(e), $8(\mathrm{j})$, and $8(\mathrm{k})$ show the appearance from the positive direction of the Z-axis. Fig. 8(c), 8(d), 8(f), 8(g), 8(h), 8(i), 8(l), and $8(\mathrm{~m})$ are from the positive direction of the $\mathrm{Z}$-axis. The dimming level is set to 2 . The light-shielding plate reflects light in the dome light in the collector mouth area, dome light in the incident mouth area, light-shielded plane mirror, light-shielded concave mirror, plane mirror, and concave mirror. The light-shielding plate does not emit light in the light-shielded conical mirror with a specular surface when $m$ is 5 , light-shielded conical mirror without a specular surface when $m$ is 5, light-shielded compound parabolic mirror, and light-shielded Fresnel lens. The plane and concave mirrors have dark areas that do not reflect light in the plane and concave mirrors. The objects in the negative direction of $\mathrm{Z}$-axis are shadows. The conical mirror, com- pound parabolic mirror, and Fresnel lens concentrate light and are bright, in the conical mirror with specular surface when $m$ is 5 , the conical mirror without specular surface when $m$ is 5 , the compound parabolic mirror, and the Fresnel lens. Therefore, obtaining the illuminance $i_{\mathrm{O}}$ by subtracting the illuminance $i_{\mathrm{S}}$ from the illuminance $i_{\mathrm{L}}$ is necessary.

To reveal the effect of reflection on the specular surface of the conical mirror, the illuminance $i_{\mathrm{O}}$ in the conical mirror with and without specular surface is measured. Figure 9 shows the measured results of the relation between the number of times $m$ and the illuminance $i_{\mathrm{O}}$. Figure 10 shows the measured results of the relation between the radius $r_{\mathrm{C}}$ and the illuminance $i_{\mathrm{O}}$. The illuminance $i_{\mathrm{O}}$ shows the case of the conical mirror with specular surface, the conical mirror without specular surface, and the specular reflection only. In the case of specular reflection only, the difference in illuminance $i_{\mathrm{O}}$ between the conical mirrors with and without a specular surface is shown. On comparing the illuminance $i_{\mathrm{O}}$ in the conical mirror with specular surface and the conical mirror without specular surface, the illuminance $i_{\mathrm{O}}$ is observed to be higher in the conical mirror with a specular surface than without a specular surface. The illuminance $i_{\mathrm{O}}$ is higher in the case of specular reflection only than in the case of the conical mirror without specular surface. Therefore, in the conical mirror, the specular reflection has a large effect on the illuminance $i_{\mathrm{O}}$. As the number of times $m$ increases, the illuminance $i_{\mathrm{O}}$ decreases. As $m$ increases, the height $h$ shown in Table 2 becomes large, and the angle $\phi_{\mathrm{R} m}$ of the light ray reaching the collector mouth shown in Tables 3 to 7 becomes small. Therefore, from the value of illuminance $i_{\mathrm{O}}$, the effect of the number $m$ and height $h$ is large and the effect of the angle $\phi_{\mathrm{R} m}$ is small. Moreover, as the radius $r_{\mathrm{C}}$ increases, the illuminance $i_{\mathrm{O}}$ decreases. As the radius $r_{\mathrm{C}}$ increases, the height $h$ and the area $a_{\mathrm{C}}$ shown in Table 2 become large. From the value of illuminance $i_{\mathrm{O}}$, the effect of height $h$ and area $a_{\mathrm{C}}$ is observed to be large. Therefore, the conical mirror with a specular surface when $m$ is 1 and radius $r_{\mathrm{C}}$ is $4.9 \mathrm{~mm}$ concentrates the most diffused light.

Table 11 shows the dimming level of the dome light with the illuminances $i_{\mathrm{C}}$ and $i_{\mathrm{I}}$. Due to the difference in area $a_{\mathrm{C}}$ and area $a_{\mathrm{I}}$, the value of illuminance $i_{\mathrm{C}}$ is higher than that of illuminance $i_{\mathrm{I}}$. Table 12 shows the measured illuminances $i_{\mathrm{S}}, i_{\mathrm{L}}$ and $i_{\mathrm{O}}$, illuminance ratio $e_{\mathrm{I}}$, and concentration ratio $e_{\mathrm{C}}$ in the measured objects. The illuminance $i_{\mathrm{L}}$ is higher than the illuminance $i_{\mathrm{S}}$. Therefore, obtaining the illuminance $i_{\mathrm{O}}$ by subtracting the illuminance $i_{\mathrm{S}}$ from the illuminance $i_{\mathrm{L}}$ is necessary. From the illuminance $i_{\mathrm{O}}$, the conical mirror concentrates the most diffused light. By using a conical mirror, it is possible to concentrate 4.36 times more light than a conventional compound parabolic mirror. From the results of Matsumoto, the illuminance ratio is 0.0039 for a polygonal mirror where 410 elliptical mirrors are arranged [19]. The conical mirror herein can concentrate 60.5 times more light compared to a polygonal mirror. Therefore, by using a conical mirror, concentrating more diffused light from the half celestial sphere is possible. The concentration ratio $e_{\mathrm{C}}$ 


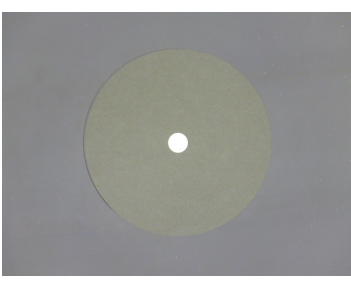

(a) Dome light in collector mouth area

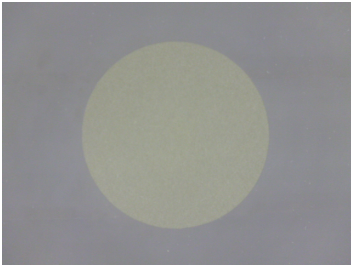

(e) Light-shielded plane mirror and light-shielded concave mirror

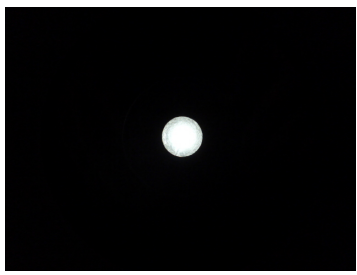

(i) Conical mirror without specular surface when $m$ is 5

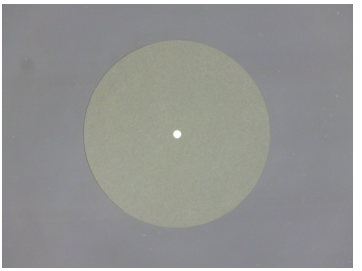

(b) Dome light in incident mouth area

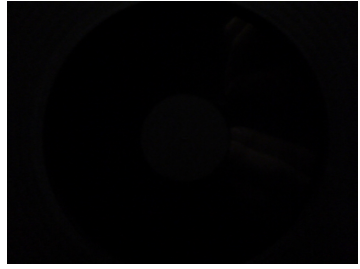

(f) Light-shielded compound parabolic mirror

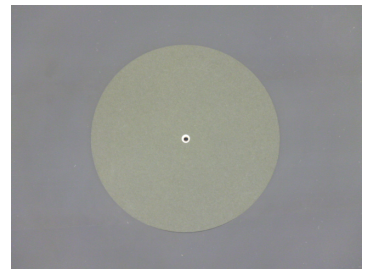

(j) Plane mirror

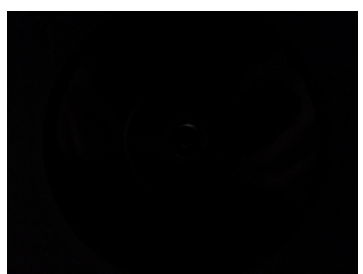

(c) Light-shielded conical mirror with specular surface when $m$ is 5

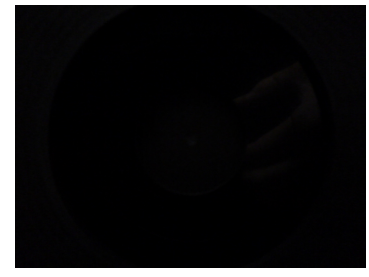

(g) Light-shielded Fresnel lens

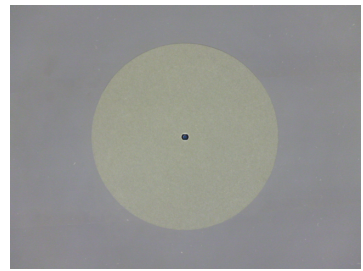

(k) Concave mirror

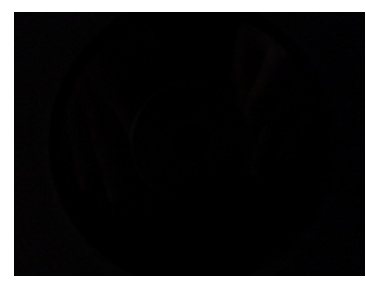

(d) Light-shielded conical mirror without specular surface when $m$ is 5

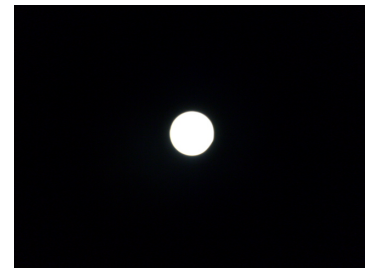

(h) Conical mirror with specular surface when $m$ is 5

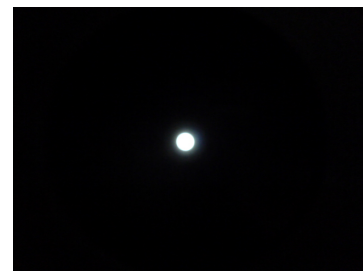

(I) Compound parabolic mirror

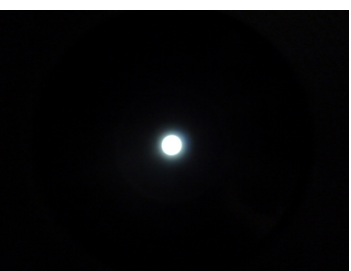

(m) Fresnel lens

Figure 8: Images of the measured objects with dome light at the collector and incident mouth areas; light-shielded conical, plane, concave, and compound parabolic mirrors and Fresnel lens; conical, plane, concave, and compound parabolic mirrors and Fresnel lens in the experimental setup.

Table 11: Comparison of the dimming level with the illuminances of the dome light in the collector mouth area and incident mouth area.

\begin{tabular}{ccc}
\hline Dimming level & $i_{\mathrm{C}}[\mathrm{lx}]$ & $i_{\mathrm{I}}[\mathrm{lx}]$ \\
\hline 2 & 1,031 & 271.7 \\
\hline
\end{tabular}

using Equation (19) shown in Table 12 and the concentration ratio $e_{\mathrm{C}}$ using Equation (15) shown in Tables 2 and 9 are compared. The difference between the two is small in the compound parabolic mirror and large in the conical mirror.

\section{Conclusion}

In this study, we designed a conical mirror that concentrates diffused light to the collector mouth from a half celestial sphere and evaluated its performance. The results obtained herein are shown below.

1. The design method was shown by establishing a coordinate system in the conical mirror. Using the design method, the actual conical mirror was manufactured from the set design values. A plane mirror, concave mirror, compound parabolic mirror, and Fresnel lens, comprising the conical mirror, are used as measured objects to compare the performance of the manufac- 
Table 12: Comparison of the illuminances of the light-shielded object, lighted object, and only the lighted object as well as the illuminance ratio and concentration ratio, in measured objects (conical, plane, concave, and compound parabolic mirrors and Fresnel lens).

\begin{tabular}{cccccc}
\hline Object name & $i_{\mathrm{S}}[\mathrm{x}]$ & $i_{\mathrm{L}}[\mathrm{x}]$ & $i_{\mathrm{O}}[\mathrm{x}]$ & $e_{\mathrm{I}}$ & $e_{\mathrm{C}}$ \\
\hline Conical mirror with specular surface when $m$ is 1 , and $r_{\mathrm{C}}$ is $4.9 \mathrm{~mm}$ & 0 & 243.7 & 243.7 & 0.236 & 1.11 \\
Plane mirror & 241.3 & 242.2 & 1.1 & 0.00107 & - \\
Concave mirror & 241.3 & 241.9 & 0.6 & 0.000582 & - \\
Compound parabolic mirror & 0.02 & 55.8 & 55.78 & 0.0541 & 4.87 \\
Fresnel lens & 0.1 & 4.26 & 4.16 & 0.00403 & - \\
\hline
\end{tabular}

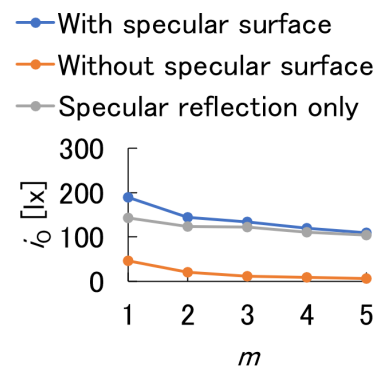

Figure 9: Measured results of the relationship between the maximum number of reflection and the illuminances of only lighted object at different surfaces and specular reflection only in conical mirror.

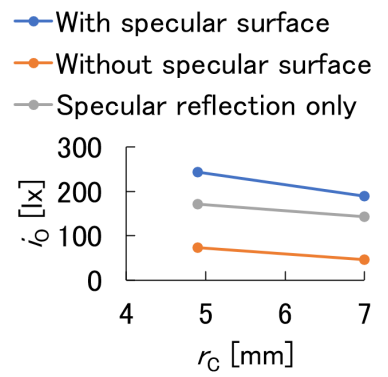

Figure 10: Measured results of the relation between the radius of collector mouth and the illuminances of only the lighted object at different surfaces and specular reflection only in the conical mirror.

tured conical mirror. An experimental setup was constructed to evaluate the performance of the measured objects. Diffused light was irradiated to the measured objects as scattering light using the constructed experimental setup. The illuminance of the light concentrated on the collector surface of the measured objects was measured for the irradiated diffused light.

2. For the conical mirror, the effect of the number of light reflections on the specular surface was revealed. The fewer the number of reflections, the greater is the illuminance in the collector mouth, and light can be concentrated. For the conical mirror, the effect of reflection on the specular surface was revealed. In the conical mirror, the effect of reflection on the specular surface is large due to the illuminance in the collec- tor mouth. Moreover, the fewer the number of reflections on the specular surface, the greater is the effect of reflection on the specular surface. For the conical mirror, the effect of the size of the collector mouth was revealed. In the conical mirror, the effect of the height and the area of the collector mouth of the conical mirror is large due to the illuminance in the collector mouth.

3. The illuminance ratio indicating the concentrating performance was 0.236 for the conical mirror and 0.0541 for the compound parabolic mirror. By using the conical mirror, it is possible to concentrate 4.36 times more light than a conventional compound parabolic mirror. By using a conical mirror, concentrating more diffused light from half celestial sphere is possible.

\section{References}

[1] Japan Solar Energy Society ed., "New Solar Energy Utilization Handbook", Japan Solar Energy Society, pp.123-128, Tokyo, 2015 (in Japanese).

[2] Japan Solar Energy Society ed., "Solar Energy Utilization Technology”, Japan Solar Energy Society, p.66, Tokyo, 2006 (in Japanese).

[3] Geospatial Information Authority of Japan ed., "National Atlas of Japan", Japan Map Center, p.47, Tokyo, 1990 (in Japanese).

[4] R. Winston, "Principles of Solar Concentrators of a Novel Design”, Solar Energy, Vol.16, No.2, pp.89-95, 1974.

[5] G. H. Derrick, I. M. Bassett and D. R. Mills, "Comparison of Reflector Designs for Stationary Tubular Solar Collectors", Solar Energy, Vol.37, No.3, pp.195-203, 1986.

[6] S. S. Rao, H. Lee and Y. Hu, "Optimal Design of Compound Parabolic Concentrator Solar Collector System”, Transactions of the ASME, Journal of Mechanical Design, Vol.136, No.9, pp.091402-1-091402-10, 2014.

[7] G. R. Mather and D. C. Beekley, "Performance of an Evacuated Tubular Collector Using Non-imaging Reflectors", Proceedings of the Joint Conference International Solar Energy Society, No.2, pp.64-78, 1976.

[8] D. R. Mills and J. E. Giutronich, "Symmetrical and Asymmetrical Ideal Cylindrical Radiation Transformers and Con- 
centrators", Journal of the Optical Society of America, Vol.69, No.2, pp.325-328, 1979.

[9] I. M. Bassett and G. H. Derrick, "Diffuse Reflectors in Nonimaging Optics", Proceedings of the International Solar Energy Society Silver Jubilee Congress, Vol.1, pp.557-560, 1979.

[10] D. P. Grimmer, "A Comparison of Compound Parabolic and Simple Parabolic Concentrating Solar Collectors", Solar Energy, Vol.22, No.1, pp.21-25, 1979.

[11] J. R. Frissora and D. M. Platt, "Drainable Evacuated Collector with Compound Parabolic Cusp Reflector", Solar Energy and Conservation, Vol.1, pp.113-140, 1980.

[12] B. Norton, P. C. Eames and Y. P. Yadav, "Symmetric and Asymmetric Linear Compound Parabolic Concentrating Solar Energy Collectors - The State-of-the-art in Optical and Thermo-physical Analysis -", International Journal of Ambient Energy, Vol.12, No.4, pp.171-190, 1991.

[13] P. C. Eames and B. Norton, "The Effect of Sky Conditions on the Partition of Incident Solar Energy between the Components of a CPC Solar Energy Collector", Proceedings of the Solar World Congress 1991 (SWC1991), Vol.2, pp.18841889, 1992.

[14] A. Suzuki, "Optimum Design for Non-imaging Solar Concentrators with Reference to Scattering Insolation Fraction", Proceedings of the Japan Solar Energy Society Japan Wind Energy Association Joint Conference, pp.37-40, 1993 (in Japanese).

[15] P. C. Eames, B. Norton, Y. Tripagnostopoulos and P. Yianoulis, "Modelling Line-axis Solar Concentrators in the Medium Temperature Range”, Renewable Energy, Vol.16, No.1, pp.743-748, 1999.

[16] J. Wang, L. Yu, C. Jiang, S. Yang and T. Liu, "Optical Analysis of Solar Collector with New V-shaped CPC", Solar Energy, Vol.135, pp.780-785, 2016.

[17] N. Yamada and Y. Honda, "Optical Analysis of High Concentrating Optical Geometry Coupled with Diffuse Solar Radiation Absorber", Journal of Japan Solar Energy Society, Vol.36, No.3, pp.45-50, 2010 (in Japanese).

[18] N. Sellami, T. K. Mallick and D. A. Mcneil, "Optical Characterisation of 3-D Static Solar Concentrator", Energy Conversion and Management, Vol.64, pp.579-586, 2012.

[19] M. Matsumoto, "Multi-part Mirror for Focusing Diffuse Light”, Mechanical Engineering Journal, Vol.4, No.3, 2017, DOI:10.1299/mej.16-00520.

[20] S. Onizuka, "Light Funnel as a Teaching Material - A Conical Device for Collecting or Projecting Light -", Journal of the Physics Education Society of Japan, Vol.42, No.1, pp.2629, 1994 (in Japanese).

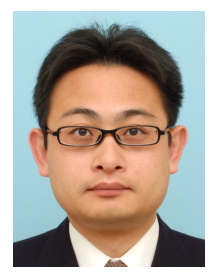

M. Matsumoto (Member) received his Ph.D. degree from University of Tsukuba. He is currently an associate professor at Kanagawa University. His current research interest is mechanical engineering. He is a member of IIAE and so on. 